\section{Wenn Hautkrebs Herpes bekommt ...} An einer Therapie des malignen Melanoms mit Hilfe onkolytischer Viren wird
seit einiger Zeit intensiv geforscht. Kann das Prinzip auch einmal zu einer
Option bei Basalzellkarzinom (BCC) und Plattenepithelkarzinom (SCC) werden?

$\mathrm{D}$ ie virale Onkolyse oder Virotherapie gilt als vielversprechender Ansatz in der Tumortherapie. Israelische Wissenschaftler züchteten nun chirurgisch gewonnene Gewebeproben von BCC und SCC in Organkultur, um daran die GewebeSelektivität zweier potenziell onkolytischer Viren, nämlich von Herpes-simplex-Viren Typ 1 (HSV-1) und Adenoviren, zu testen. Beide Tumorgewebe, BCC wie SCC, erwiesen sich als empfindlich gegenüber einer Infektion mit diesen Viren.

Nach Inkubation der Kulturen mit HSV1 konnte nach 7 Tagen immunhistologisch eine extensive, durch die Viren induzierte Zytolyse nachgewiesen werden. Die weitere Charakterisierung der infizierten Zellen ergab, dass die Herpesviren eine spezielle Subpopulation früher Vorläuferzellen des BCC- bzw. SCC-Gewebes angreifen: Die infizierten Zellen waren positiv für den Transkriptionsfaktor p63 und negativ für die Keratinozyten-Marker K14 und K15.

Die Ergebnisse sind vor dem Hintergrund des gegenwärtigen Verständnisses der Entstehung heller Hauttumoren zu sehen: Danach fungieren Haarwurzelscheiden als Reservoir für Haut-Stammzellen („Stammzellnischen“), die für die Regeneration und Re-Epithelialisierung im Rahmen der Wundheilung zuständig sind. Während der langen Lebensspanne der Stammzellen akkumulieren diese somatische Mutationen, die im ungünstigen Fall zur Deregulation der normalen Prozesse bei der Wundheilung führen können - und in der Folge zur Tumorbildung.

Neben Tumorzellen, die charakteristische Marker von Haarfollikelzellen (K15) trugen, identifizierten die Autoren weitere Basalzellen im BCC- und im SCCGewebe mit dem Merkmal p63. Diese Zellen könnten Tumorvorläuferzellen sein; interessanterweise wurden sie bevorzugt durch HSV-1 infiziert.

Fazit: Den Autoren zufolge könnten diese Befunde den Weg zu einer zukünftigen Nutzung des Herpesvirus als AntitumorAgens weisen: Die Eradikation von Tumorstamm- bzw. Tumorvorläuferzellen dürfte ein entscheidender Schritt für die Verhinderung von Rezidiven sein.

Waltraud Paukstadt

Hochberg M et al. Tropism of herpes simplex virus type 1 to nonmelanoma skin cancers. Br J Dermatol 2011; 164: 273-81

\title{
Chronisches Handekzem: Was tun beim Rezidiv?
}

Für Patienten mit therapierefraktärem chronischem Handekzem bietet Alitretinoin seit zwei Jahren eine Behandlungsoption. Wie effektiv ist diese Therapie bei erneuter Symptomverschlechterung?

A litretinoin, ein Vitamin-A-Derivat, greift inhibierend in verschiedene Entzündungsprozesse ein. In der BACHStudie (Benefit of Alitretinoin in Chronic Hand Ekzema) erwies sich die systemische Gabe dieser Substanz bei chronischem Handekzem, das auf eine lokale Glukokortikoidtherapie nicht bzw. ungenügend ansprach, als wirksame Therapie. Für diese Indikation ist Alitretinoin in mehreren europäischen Ländern bereits zugelassen.

In einer aktuellen Studie wurden 117 Patienten mit chronischem Handekzem aufgenommen, die in der BACH-Studie auf die Behandlung gut angesprochen hatten und von den Ärzten als „frei“ oder "fast frei“"von Ekzem beurteilt worden waren. Bei ihnen war es innerhalb von 24 Wochen zum Rezidiv gekommen - in dieser Zeit waren als Behandlung nur Emollienzien zugelassen. Die Studienteilnehmer erhielten randomisiert einmal täglich entweder ihre frühere Studienme- dikation oder Plazebo (30 mg Alitretinoin vs. Placebo, $10 \mathrm{mg}$ Alitretinoin vs. Placebo) für 12-24 Wochen. Als erneutes Ansprechen war die Einstufung als „frei“ oder „fast frei“ von Läsionen definiert.

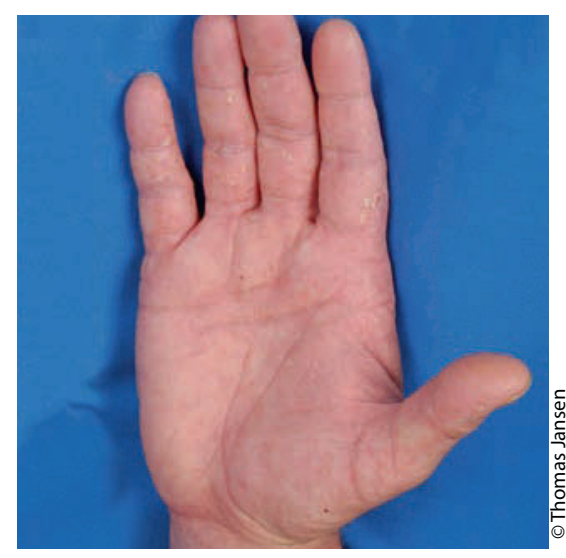

Konnte ein Handekzem durch Alitretinoin zur Abheilung gebracht werden, so ist bei einem Rezidiv ein erneuter Versuch durchaus gerechtfertigt.
In der 30-mg-Gruppe zeigte sich eine gegenüber Plazebo signifikant erhöhte Ansprechrate ( $80 \%$ vs. $8 \%, \mathrm{p}<0,001)$. Unter der Gabe von $10 \mathrm{mg}$ Alitretinoin sprachen $48 \%$ der Studienteilnehmer an - gegenüber $10 \%$ unter Plazebo. Die Abnahme im modifizierten Total Lesion Symptom Score (mTLSS) lag nach 24 Wochen unter $30 \mathrm{mg}$ Alitretinoin bei $92 \%$, unter $10 \mathrm{mg}$ bei $71 \%$ und in der Plazebogruppe bei $43 \%$. An unerwünschten Wirkungen waren unter $30 \mathrm{mg}$ Alitretinoin Kopfschmerzen am häufigsten. Andere Nebenwirkungen traten nur geringfügig häufiger auf, als unter Plazebo.

Fazit: Die Autoren schließen aus den Ergebnissen, dass die Behandlung eines bisher therapierefraktären chronischen Handekzems mit Alitretinoin bei Respondern auch im Rezidivfall effektiv und gut verträglich ist. In diesen Fällen eignet sich die unterbrochene Behandlung mit diesem Wirkstoff auch zum Langzeitmanagement der Erkrankung.

Andreas Fischer

Bissonnette R et al. Successful retreatment with alitretinoin in patients with relapsed chronic hand eczema. Br J Dermatol 2010; 162: 420-6 